\title{
Influence of Woody Breast Myopathy on Sarcomere Length and Tensile Strength in Commercial Broiler Pectoralis major Muscle
}

\author{
T. Eero J. Puolanne*, Cristina G. Costandache, and Per Ertbjerg \\ Department of Food and Nutrition, University of Helsinki, Fl-00014 Helsinki, Finland \\ *Corresponding author. Email: eero.puolanne@helsinki.fi (T. Eero J. Puolanne)
}

\begin{abstract}
Woody breast syndrome is characterized by degenerative changes at the muscle fiber level and accumulation of connective tissue between the fibers. This study explored effects of the syndrome on muscle properties by focusing on a comparison of the sarcomere lengths between normal and woody breast muscles, including cranial and middle parts, surface and deeper layers, electrically stimulated and nonstimulated muscles, and their combinations. Tensile strengths longitudinally and transversally to the muscle fiber direction in the cranial and middle parts of the muscles were determined. The overall sarcomere lengths of woody breasts were longer than normal muscles $(1.93 \mu \mathrm{m}$ vs. $1.88 \mu \mathrm{m} ; P<0.05)$. The surface layer had overall longer sarcomere lengths than the deeper layer (cranial surface vs. cranial deeper layer: $1.97 \mathrm{vs.}$ $1.89 \mu \mathrm{m}$; middle surface vs. middle deeper layer: 1.93 vs. $1.84 \mu \mathrm{m} ; P<0.05)$. Compared with normal breast muscles, woody breast muscles had longer sarcomeres in the surface layer; however, sarcomere length did not differ in the deeper layer. Electrically stimulated chicken breasts generally had longer sarcomere lengths $(2.00$ vs. $1.82 \mu \mathrm{m} ; P<0.001)$. There was no significant difference in tensile strength between normal and focal woody breast (mild local lesion, usually in the cranial end) samples when fiber direction or sample location was studied $(P>0.05)$, but there was a clear difference between normal and focal versus diffuse (severe woody breast lesion throughout the muscle) samples $(P<0.001)$. Tensile strength was much greater in diffuse woody breast muscles when extended longitudinally or transversely to the fiber direction. In conclusion, although this study did not show sarcomere lengths in living muscle, it suggests an imbalance in sarcomere lengths in different parts of the breast muscle, which may induce a reduction in the functionality and strength of the muscle.
\end{abstract}

Key words: broiler breast myopathy, muscle contraction, mechanical strength, electrical stimulation, sampling location Meat and Muscle Biology 5(1): 12,1-11 (2021) doi:10.22175/mmb.11564

Submitted 14 July 2020

Accepted 16 December 2020

\section{Introduction}

Woody breast syndrome (Sihvo et al., 2014) is a relatively new degenerative disease in broiler breast muscle that affects the Pectoralis major muscle and often coexists with another emerging breast muscle syndrome, white striping (Kuttappan et al., 2013). The mechanical removal of breast muscle at about $4 \mathrm{~h}$ postmortem may reveal a third syndrome called "spaghetti meat," which is characterized by a separation of muscle fiber bundles (Petracci et al., 2019). The great significance of these syndromes, which cause marked quality and possibly also animal welfare problems (Norring et al., 2019), has resulted in many published papers during the last 6 to $8 \mathrm{y}$. All of the 3 syndromes may coexist, which suggests that they may have the same triggering causes (Petracci et al., 2019), but further research on mechanisms is still needed. Possible risks in animal welfare have also motivated more fundamental research to identify the mechanisms of the syndromes to alleviate the problems (Norring et al., 2019). Woody breast syndrome causes degenerative changes at the muscle fiber level as well as accumulation of connective 
tissue materials between the fibers and on the surface of the muscles (Sihvo et al., 2014). The texture of raw woody breast meat is characterized by hardness (Sihvo et al., 2014; Soglia et al., 2017), especially in the surface layer of the muscles and where the lesion has already started at earlier ages. Most hypotheses for the mechanism of woody breast formation stress poor blood circulation in the breast muscle due to the larger size of the birds and/or fillets (Sihvo et al., 2014; Abasht et al., 2016; Soglia et al., 2016; Baldi et al., 2018; Sihvo et al., 2018), phlebitis (Papah et al., 2017), low capillarity (Sihvo et al., 2018), or hypoxia (Petracci et al., 2019).

The purpose of this study was to obtain basic information on sarcomere length and tensile strength of muscle structures within poultry woody breast, and in particular to discuss structural aspects of the damage of muscle fibers and/or fiber bundles seen histologically (Sihvo et al., 2014) and at the macroscopic level. The operational aims were to study the sarcomere lengths in different breast muscle locations and the tensile strength of muscle tissue, longitudinally and transversely to the fiber direction. In addition, the effect of electrical stimulation on sarcomere lengths was studied.

\section{Materials and Methods}

\section{Sample collection and handling}

In this study, 2 individual experiments were performed, one for measurement of sarcomere lengths and one for tensile tests. There were 2 sampling sessions for each of the experiments. The birds were slaughtered at 2 Finnish commercial slaughterhouses, Atria Finland Plc (Seinäjoki, Finland) and HKScan Co (Eura, Finland). Both slaughterhouses used $\mathrm{CO}_{2}$ stunning, and the stunning methods, cooling programs, and cutting line schedules were essentially similar. Carcasses were cooled by normal industrial cooling systems, and breast muscles were excised on-line $4 \mathrm{~h}$ postmortem. The selection of the chicken breasts was made based on visual evaluation and palpation by 2 experienced persons, according to the method by Sihvo et al. (2014). All the breasts were from 37-dold Ross 308 hybrid birds of both sexes with an average carcass weight of $1.6 \mathrm{~kg}$ (Aviagen UK Ltd, Midlothian, Scotland). The birds were from the same hatchery. All birds within a sampling were from the same farm, but different samplings were from different farms. Feeding was done at all farms according to the instructions of Aviagen Ltd.

\section{Sarcomere length measurement}

For the sarcomere length study, 20 normal (unaffected) breasts (mean weight $174 \mathrm{~g}$ ) and 21 woody breasts fillets $(262 \mathrm{~g})$ were collected. Ten normal breasts were collected from each slaughterhouse and 10 to 11 woody breasts. For sarcomere length studies, only 2 categories were used: the normal breasts showed no color defects, slime, or palpatable hardness, and the woody breast samples showed a clear focal lesion that was not, however, found in the deep layers. Electrical stimulation was applied after slaughter only at one slaughterhouse, and the effect was therefore confounded with slaughter facility effects. After transportation from the slaughterhouses in Styrofoam boxes with frozen packs for cooling, the chicken breasts were stored in individual plastic bags overnight at a temperature of $4^{\circ} \mathrm{C}$. The following day, all the samples were weighed. The breasts were either immediately prepared for sarcomere length measurement or stored frozen at $-20^{\circ} \mathrm{C}$ for up to $24 \mathrm{~d}$ before the analyses.

Sarcomere lengths were determined according to Cross et al. (1981) with minor modifications (Liu et al., 2014). Samples were taken from 4 different locations from each breast: the cranial and middle area (middle being the center along the cranial-caudal axis of the muscle) and the surface layer and deeper layer. Approximately $1 \mathrm{~g}$ of meat was excised from each of the mentioned locations in the chicken breasts. In order to fix the sarcomere length, $10 \mathrm{~mL}$ formalin solution (3.5\% formaldehyde in $0.085 \mathrm{M}$ phosphate buffer) was added. The mix was homogenized with an NSI12 homogenizer (Bodine Electric Company, Chicago, IL). A drop of homogenate was smeared on a glass slide and passed under Novette 1507-0 Helium-neon gas laser (Uniphase, Manteca, CA) (Cross et al., 1981). The distance between the slide holder and the screen was measured. The laser beam was refracting from the sarcomeres on the slide and created a light pattern of 3 longitudinal lines. The distance from the central line and one of the extremes was measured, and the value was used in the formula from Cross et al. (1981) to determine the sarcomere length. Mean values were calculated from 25 measurements of each homogenate.

\section{Tensile test}

The tensile test study sampling involved chicken breasts only from the slaughterhouse not using electrical stimulation. The samples were selected based on the degree that they were affected by the woody breast condition. For tensile strength studies, 3 experimental 
groups were defined: normal (unaffected), focal (with mild local woody breast lesion, usually in the cranial end of the muscle), and diffuse (with severe woody breast lesion throughout the muscle) (Sihvo et al., 2014).

In the first sampling session for tensile tests, 20 normal chicken breasts were used, together with 10 focal cases and 10 diffuse cases. In a second sampling, another 10 focal and 10 diffuse breasts were collected, resulting in a total of 20 samples in each group. After transportation from the slaughterhouse, the chicken breasts were stored overnight at $4{ }^{\circ} \mathrm{C}$ and weighed the next day, and sample pieces were prepared and kept at $4^{\circ} \mathrm{C}$ for the tensile tests, which were performed on day 3 postmortem.

Samples were cut using a metal mold-from a slice $6 \mathrm{~mm}$ thick from the surface layer only_of the cranial and middle area of every chicken breast, both longitudinal and transverse to the direction of the muscle fibers (Figure 1). The longitudinal tensile strength primarily reflects the strength of structures within the muscle fibers and fiber bundles, whereas the transverse direction more reflects the strength of connective tissue in the extracellular matrix holding the fibers and fiber bundles together. It was possible to take only 1 test piece from each breast/location. A template was used to obtain the dumb-bell shape of the samples. Length and thickness varied among samples, but the width of the central point was constant $(6 \mathrm{~mm})$. Samples were pulled with an Instron Universal Testing Machine (Instron Corporation, Norwood, MA), with a static load cell of $100 \mathrm{~N}$, at a speed of $50 \mathrm{~mm} / \mathrm{min}$. In order to provide a better grip of the sample, duct tape with a rough surface was used on the interior side of the clamps.

\section{Statistical analyses}

Statistical analysis was done with SAS Enterprise Guide 5.1 software (SAS Institute Inc., Cary, NC). The breast muscles in sarcomere length and tensile strength studies were different, and therefore the datasets from these experiments were studied separately, using the analysis of variance. Data were analyzed using the General Linear Model procedure of SAS with each breast muscle being an experimental unit. For sarcomere length, the effects of woody breast, location, and electrical stimulation as well as the interactions between them were tested. For tensile strength, the effects of woody breast severity on the main effects of fiber direction, sample location, and their interactions were tested.

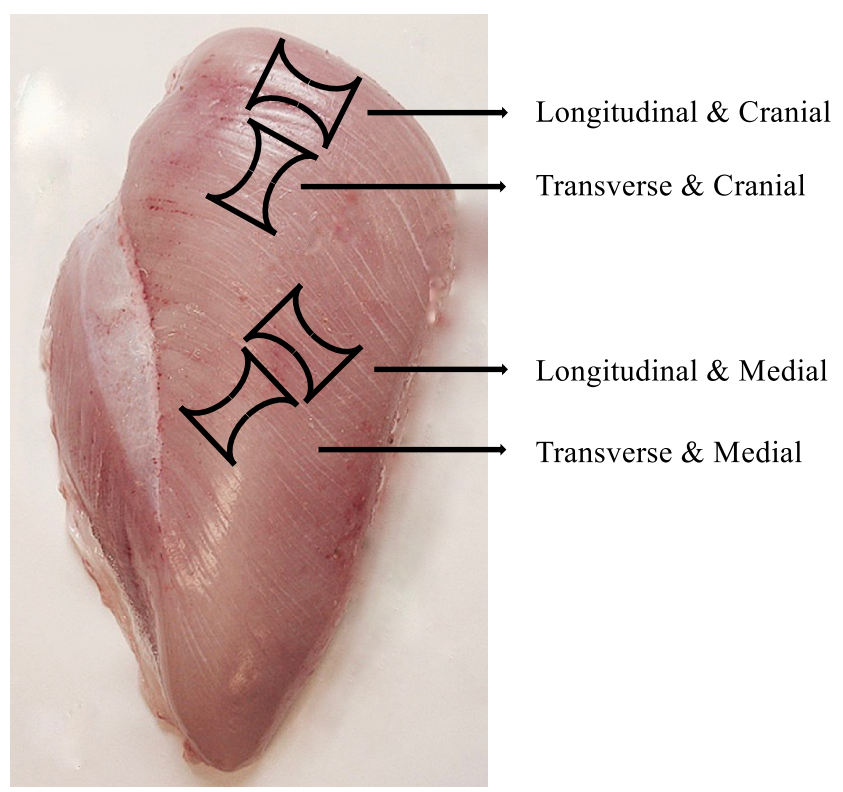

Figure 1. Chicken breast fillet sampling sites for the tensile tests showing the dumb-bell shape in relation to muscle extension direction (longitudinal and transverse to fiber direction) and Pectoralis major muscle location (cranial and middle area).

\section{Results}

\section{Sarcomere length}

The woody breast condition, location within the muscle, and electrical stimulation all showed significant effects on sarcomere length as well as 3 significant interactions (Table 1). Analysis of main effects showed slightly longer $(P<0.05)$ sarcomere lengths in woody breast compared with normal samples, in surface compared with deep samples, and in electrical stimulated compared with nonstimulated samples. However, the interactions of woody breast and electrical stimulation and electrical stimulation and location showed that the longer sarcomere lengths from electrical stimulation negated the woody breast and location effects on sarcomere length. Furthermore, the woody breast and location interaction indicated that the woody breastaffected muscles had longer sarcomeres compared with the normal ones in the surface layer (both cranial and middle areas), whereas the sarcomere lengths in the deep layer did not differ (Figure 2).

\section{Tensile strength}

Woody breast condition, fiber direction, and sample location within the muscle all affected the tensile strength of the muscle strips, as did the 2-way interactions of location and woody breast, direction and woody breast, and location and direction (Table 2). 
Table 1. Sarcomere lengths as affected by WB condition, muscle location (cranial/middle, surface/ depth), and electrical stimulation

\begin{tabular}{llcccc}
\hline \hline \multirow{2}{*}{ Effect } & \multicolumn{1}{c}{ Category } & LS & & & \\
\hline WB & Normal $(\mu \mathrm{m})$ & SEM & $P$ & $N$ \\
\hline \multirow{2}{*}{ Location } & WB & $1.88^{\mathrm{a}}$ & 0.01 & $<0.05$ & 80 \\
& CS & $1.93^{\mathrm{b}}$ & & & 84 \\
& CD & $1.97^{\mathrm{c}}$ & 0.02 & $<0.05$ & 41 \\
& MS & $1.89^{\mathrm{ab}}$ & & & 41 \\
ES & MD & $1.93^{\mathrm{bc}}$ & & & 41 \\
& NES & $1.84^{\mathrm{a}}$ & & & 41 \\
WB and & NS & $1.82^{\mathrm{a}}$ & 0.01 & $<0.0001$ & 84 \\
ES & Normal and NES & $2.00^{\mathrm{b}}$ & & & 80 \\
& & $1.77^{\mathrm{a}}$ & 0.02 & $<0.001$ & 40 \\
& Normal and ES & $1.99^{\mathrm{c}}$ & & & \\
& WB and NES & $1.87^{\mathrm{b}}$ & & & 40 \\
& WB and ES & $2.00^{\mathrm{c}}$ & & & 44 \\
ES and & NES and CS & $1.91^{\mathrm{b}}$ & 0.02 & $<0.05$ & 21 \\
Location & & & & & \\
& NES and CD & $1.75^{\mathrm{a}}$ & & & 21 \\
& NES and MS & $1.90^{\mathrm{b}}$ & & & 21 \\
& NES and MD & $1.71^{\mathrm{a}}$ & & & 21 \\
& ES and CS & $2.03^{\mathrm{c}}$ & & & 20 \\
& ES and CD & $2.03^{\mathrm{c}}$ & & & 20 \\
& ES and MS & $1.96^{\mathrm{bc}}$ & & & 20 \\
& ES and MD & $1.96^{\mathrm{bc}}$ & & & 20 \\
\hline
\end{tabular}

${ }^{\mathrm{a}-\mathrm{c}}$ Means within each effect with the same letter do not differ $(P>0.05)$.

$\mathrm{CD}$, cranial depth; CS, cranial surface; ES, electrical stimulation; LS, least-squares; MD, middle depth; MS, middle surface; NES, no electrical stimulation; SEM, standard error of the mean; WB, woody breast.

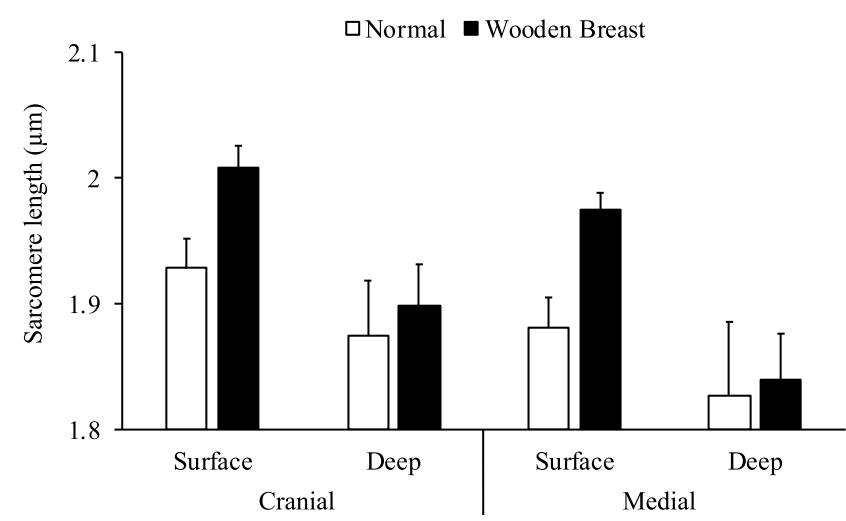

Figure 2. Sarcomere lengths of normal and woody breast muscles in the surface and deep layer of the cranial and middle area. Bars show the standard error $(N=20$ normal/21 woody breast).

The normal group and focal group did not differ in tensile strength, neither when fiber direction nor when sample location was taken into account $(P>0.05)$. However, there was a clear difference between the normal and focal groups versus the diffuse group (Table 2;
$P<0.001)$ : there was a dramatic increase of tensile strength in the diffuse group compared with the normal and focal groups (Table 2 and Figure 3).

The longitudinal stretching direction of the muscle showed significantly higher tensile strength compared with the transverse direction $(P<0.001)$. Regardless of cranial or median location, samples stretched in the longitudinal direction of fibers yielded higher values of tensile strength compared with the transverse direction $(P<0.05)$. This difference was even more obvious in the diffuse samples. Location alone was not a

Table 2. Tensile strength values as affected by WB condition, fiber direction, and muscle location (cranial area and middle area) of the chicken breast muscles

\begin{tabular}{|c|c|c|c|c|c|}
\hline Effect & Category & $\begin{array}{c}\text { LS } \\
\text { Mean }(\mathrm{N})\end{array}$ & SEM & $P$ & $N$ \\
\hline \multirow[t]{3}{*}{ WB } & Normal & $3.71^{\mathrm{a}}$ & 0.32 & $<0.0001$ & 80 \\
\hline & Focal & $3.64^{\mathrm{a}}$ & & & 80 \\
\hline & Diffuse & $9.16^{\mathrm{b}}$ & & & 80 \\
\hline \multirow[t]{2}{*}{ Direction } & Longitudinal & $6.84^{\mathrm{b}}$ & 0.25 & $<0.0001$ & 120 \\
\hline & Transverse & $4.17^{\mathrm{a}}$ & & & 120 \\
\hline \multirow[t]{6}{*}{$\begin{array}{l}\text { Location } \\
\text { and WB }\end{array}$} & $\begin{array}{l}\text { Cranial and } \\
\text { normal }\end{array}$ & $3.17^{\mathrm{a}}$ & 0.44 & $<0.0001$ & 40 \\
\hline & Cranial and focal & $3.09^{\mathrm{a}}$ & & & 40 \\
\hline & $\begin{array}{l}\text { Cranial and } \\
\text { diffuse }\end{array}$ & $9.82^{\mathrm{b}}$ & & & 40 \\
\hline & $\begin{array}{l}\text { Middle and } \\
\text { normal }\end{array}$ & $4.25^{\mathrm{a}}$ & & & 40 \\
\hline & Middle and focal & $4.20^{\mathrm{a}}$ & & & 40 \\
\hline & $\begin{array}{l}\text { Middle and } \\
\text { diffuse }\end{array}$ & $8.52^{\mathrm{b}}$ & & & 40 \\
\hline \multirow[t]{6}{*}{$\begin{array}{l}\text { Direction } \\
\text { and WB }\end{array}$} & $\begin{array}{l}\text { Longitudinal and } \\
\text { normal }\end{array}$ & $4.30^{\mathrm{ab}}$ & 0.44 & $<0.01$ & 40 \\
\hline & $\begin{array}{l}\text { Longitudinal and } \\
\text { focal }\end{array}$ & $3.90^{\mathrm{ab}}$ & & & 40 \\
\hline & $\begin{array}{l}\text { Longitudinal and } \\
\text { diffuse }\end{array}$ & $12.32^{\mathrm{c}}$ & & & 40 \\
\hline & $\begin{array}{l}\text { Transverse and } \\
\text { normal }\end{array}$ & $3.11^{\mathrm{a}}$ & & & 40 \\
\hline & $\begin{array}{l}\text { Transverse and } \\
\text { focal }\end{array}$ & $3.39^{\mathrm{a}}$ & & & 40 \\
\hline & $\begin{array}{l}\text { Transverse and } \\
\text { diffuse }\end{array}$ & $6.02^{\mathrm{b}}$ & & & 40 \\
\hline \multirow[t]{4}{*}{$\begin{array}{l}\text { Location } \\
\text { and direction }\end{array}$} & $\begin{array}{l}\text { Cranial and } \\
\text { longitudinal }\end{array}$ & $7.27^{\mathrm{c}}$ & 0.35 & $<0.05$ & 60 \\
\hline & $\begin{array}{l}\text { Cranial and } \\
\text { transverse }\end{array}$ & $3.45^{\mathrm{a}}$ & & & 60 \\
\hline & $\begin{array}{l}\text { Middle and } \\
\text { longitudinal }\end{array}$ & $6.41^{\mathrm{c}}$ & & & 60 \\
\hline & $\begin{array}{l}\text { Middle and } \\
\text { transverse }\end{array}$ & $4.90^{\mathrm{b}}$ & & & 60 \\
\hline
\end{tabular}

a-cMeans within each effect with the same letter do not differ $(P>0.05)$. LS, least-squares; SEM, standard error of the mean; WB, woody breast. 


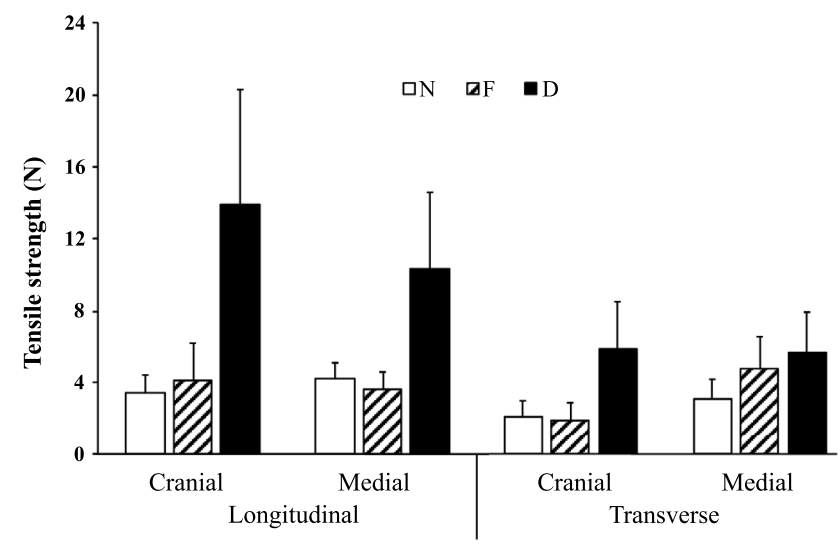

Figure 3. Tensile strength of 2 muscle extension directions (longitudinal and transverse to muscle fiber direction) in the cranial and the middle area of normal, focal, and diffuse samples. Bars show the standard error $(N=20)$.

significant factor regarding the tensile strength (results not shown), but the interaction between location and the 3 experimental groups proved to be highly significant $(P<0.0001)$. The recorded maximum load had the highest mean value in diffuse samples, both in the cranial area and in the middle area of the chicken breasts.

\section{Discussion}

\section{Sarcomere length}

As the position of wings postmortem determines the degree of the contraction of sarcomeres (Williams and Goldspink, 1978), sarcomere lengths vary in breast muscles. The real sarcomere lengths in the resting breast muscles of living birds is, to our knowledge, not known. At commercial slaughter, the wings are folded about mid-way down when the muscles enter rigor mortis in the cooling line, which influences the final sarcomere length in rigor muscles. In the present study, the mean sarcomere length in normal chicken breast was 1.77 $\mu \mathrm{m}$ when electrical stimulation was not used and 1.99 $\mu \mathrm{m}$ when electrically stimulated. The sarcomere length of the nonstimulated group is similar to the values recorded by Ashmore et al. (1988), Kang and Sams (1999), and Soglia et al. (2019). Sun at al. (2018) found shorter sarcomere lengths ( $1.66 \mu \mathrm{m}$ for normal), but they excised the muscles $2 \mathrm{~h}$ post-slaughter, and therefore the muscles may have shortened more than muscles still attached to the skeleton.

Some poultry slaughterhouses use electrical stimulation to reduce the risk of cold-induced shortening during cooling and after the subsequent cutting, i.e., induced shortening. In general, electrical stimulation affected normal muscles to a larger degree than the woody breasts, and the deep layer to a larger degree than the surface layer. The woody breast condition has a significant effect on sarcomere length $(P<$ $0.05)$, as the results of the current study show overall longer sarcomere lengths in woody breast-affected samples $(1.87 \mu \mathrm{m})$ when electrical stimulation is not used. This is in agreement with the findings of Tijare et al. (2016), who measured sarcomere lengths of non-electrically stimulated breast muscles affected by woody breast and white striping. In the study by Tijare et al., the average sarcomere length was $1.80 \mu \mathrm{m}$ in the most severe cases, which is slightly shorter than the $1.87 \mu \mathrm{m}$ measured in nonstimulated woody breast samples in the current study. Sun et al. (2018) reported a length of $1.82 \mu \mathrm{m}$ for severe woody breast. On the contrary, Velleman et al. (2018) found longer sarcomeres in unaffected samples compared with woody breast samples. Their samples were fixed after the excision of the muscles after the slaughter process, to avoid further contraction. In their study, the sarcomere lengths of unaffected samples were longer than in this study. The present study shows that sarcomere length is longer in the surface layer. In most studies, however, the depth of the sampling site has not been given, which makes the comparisons unreliable.

Ashmore et al. (1988) showed that, in dystrophic chicken breast muscles, the sarcomere lengths were longer compared with those of normal muscles. Muscular dystrophies are a group of genetic diseases characterized by gradual weakness and loss of muscle mass. Also, woody breast expresses myopathy features such as decreased number of myofibers, increased diameter of myofibers, necrosis, and fibrosis (Sihvo et al., 2014). The reason for the difference in sarcomere lengths was not specifically given by Ashmore et al. (1988), but it was suggested that dystrophic muscle fibers are exposed to increased passive tension during the initial growth phases. Consequently, it is also assumed that the increased growth rate in chickens with wooden breast myopathy could induce the muscle fibers to be under more stretch than those in normal muscle.

Liu et al. (2020) showed that single muscle fibers, isolated from the non-lesion area of focal woody breast muscle, have a relaxed (slack) sarcomere length of $1.95 \mu \mathrm{m}$, an about $40 \%$ larger cross sectional area compared with normal muscles with the sarcomere length of $2.03 \mu \mathrm{m}$ (i.e., the difference was $4 \%$ ). These results show an opposite trend as found in the current study; however, the result of Liu et al. was calculated on the basis of slack length in live muscle fiber simulation, whereas in the current study the values are for the actual length in the muscle after completion of rigor. In the 
present study, the overall sarcomere length of normal muscles was $1.88 \mu \mathrm{m}$ and that of woody breast muscles $1.93 \mu \mathrm{m}$. Therefore, if the postmortem contraction of the normal muscles is greater than that of the woody breast muscles, this may indicate some kind of dysfunction of woody breast muscle fibers.

In the current study, both the woody breast and normal breast samples from electrically stimulated carcasses had longer sarcomere length compared with those in which electrical current was not applied $(P<0.0001)$. This was an expected outcome because the purpose of electrical stimulation is to eliminate cold-induced shortening during cooling as well as shortening due to early deboning and thus improve tenderness (Sams and Dzuik, 1999; Ertbjerg and Puolanne, 2017). Therefore, it is important to notice that the shortest sarcomeres were in nonstimulated normal muscles and that the nonstimulated woody breast muscles had shorter sarcomeres than stimulated, suggesting that some induced shortening occurred in nonstimulated muscles, although this shortening was less extensive in woody breast. Woody breast muscles are known to have less glycogen and higher ultimate $\mathrm{pH}$ than normal (Dalle Zotte et al., 2014; Petracci et al., 2019), and this can explain the decreased induced shortening in woody breast. In agreement, electrical stimulation resulted in 7\% longer sarcomeres in woody breast muscles $(P<0.05)$ compared with nonstimulated muscles, whereas the difference between the normal chicken breasts was $12 \%$. The results may suggest that the deeper muscle layer is more prone to cold shortening and that woody breast is less capable of shortening than normal, especially in the surface layer possibly because of structural damages.

The force and velocity that can be developed in skeletal muscle is linked with the sarcomere length (Gordon et al., 1966), and the evaluation of sarcomere length can supply valuable information not only regarding the architectural design of the muscle but also about the textural properties of the muscle. According to Biewener (2011), the Pectoralis muscle has a special property of being able to create force over a long contraction distance and shorten $33 \%-42 \%$ of the resting fiber length. This is necessary because the connection to the humerus (Biewener, 2011) necessitates a wide contraction range with full power. At rest, the wings of living chicken are mostly folded down, i.e., Pectoralis major sarcomeres are shortened.

Furthermore, it can be assumed that a part of the difference (longer sarcomeres in woody breast) may have been caused by structural damages at the microstructure level resulting in a less efficient force transmission during contraction. It has been shown that lateral force transmission is impaired in dystrophic mice, due to disruptions in the connections between muscle fiber cytoskeleton and the extracellular matrix (Ramaswamy et al., 2011). When Ashmore et al. (1988) examined sarcomere lengths in stretched and non-stretched Patagialis muscles of normal and dystrophic chicken, they found a higher increase in sarcomere length-due to the artificially induced stretching the Patagialis muscles were subjected to-in normal compared with dystrophic muscles (44\% and 25\%, respectively), indicating an increased stiffness in dystrophic muscles. With single fiber studies, Liu et al. (2020) found that the force generated by fibers isolated from the non-lesion area of focal woody breast muscles was higher than normal fibers at higher stretching levels, but when the $20 \%$ higher diameter of woody breast sample fibers was shrunk osmotically to the same level as normal fibers, the difference disappeared.

It has previously been shown that there is a gradual decrease of histopathological lesions in woody breast from the surface to the deeper layer of the muscle (Soglia et al., 2016, 2017), suggesting that the severity of this myopathy is higher in the surface layer. The mean sarcomere lengths of the samples from the surface layer were approximately $8 \%$ longer in woody breast muscles compared with normal muscles, and in the deeper layer, the difference between the woody breast samples and the normal samples was $11 \%$, indicating a trend of looser structure inside woody breast muscles. Further research regarding the relationship between the woody breast condition and the location within the muscle could contribute to the understanding of their interaction-e.g., studying sarcomeres in different parts of the muscle, because the maximum force in different sites of the living muscle differ (Liu et al., 2020)_and it is, in theory, possible that there will be tensions within the contracting muscles. It must be remembered that muscles are not elastic when contracted with maximal force, and the fastglycolyzing fibers of Pectoralis muscles contract simultaneously.

In the current study, the chicken breasts collected from slaughterhouses were stored overnight; a part of the samples were prepared for measurements the following day, and the second part were freeze stored at $-20^{\circ} \mathrm{C}$ for $24 \mathrm{~d}$ before preparation. According to the literature, the rigor phase takes place in the first $5 \mathrm{~h}$ postmortem, with different onset times in cranial (first) and caudal (later) regions of the Pectoralis muscle (Papa and Fletcher 1988; Li et al., 2010). It is clear that the chicken muscles used for our study had reached the rigor phase, after which no changes in sarcomere 
lengths will take place. However, there was a very clear difference between the mean weights of normal and woody breasts: 174 versus $262 \mathrm{~g}$. It is known that woody breasts are heavier (Petracci et al., 2019) than normal ones, partly due to higher growth of the muscles as well as the birds and partly because there is extracellular material in woody breast muscles. Therefore, it is not possible to conclude what the primary reason for the higher weight of woody breasts is because several factors-such as genetic variation, feeding, fibrosis, and edema-may contribute to the ultimate weight and may eventually contribute to some extent to the formation of the syndrome.

\section{Tensile strength}

The tensile tests were done to obtain basic knowledge for the physical strength of woody breast and normal breast muscles and compare that with the tension created in muscle shortening, using living muscle shortening force data from the literature. It should be mentioned that the samples were taken from the distal surface, i.e., skin side, where the woody breast lesion is usually most severe. The results of our experiment showed no significant difference between the mean values of tensile strength in normal samples and focal samples but significantly higher values in diffuse woody breast samples $(P<0.0001)$. It was rather unexpected that the tensile strength was not significantly different between the normal samples and focal samples taken from the cranial area, since all the chicken breasts with focal lesion presented woody breast characteristics in the cranial area. As woody breast usually starts focally and the severity is a continuum, a possibility could be that, in the early stages of woody breast development, the tensile properties of the chicken breasts were not yet affected to a significant extent at the tensile test sampling locations.

Some texture characteristics of raw and cooked chicken breasts with woody breast myopathy were studied by Chatterjee et al. (2016). They reported increased shear force and hardness of raw woody breast fillets compared with normal chicken breasts, in agreement with the increased tensile strength of woody breast samples compared with normal samples found in our study. In the study by Chatterjee et al. (2016), the chicken breast samples were also grouped based on the severity of woody breast, and in most of the measurements, the samples from the moderate group had significantly higher values than the normal group but not significantly lower mean values than the samples from the severe group. This discrepancy between the study Chatterjee et al. (2016) study and the current study regarding the relationship between groups with different severity levels of woody breast could be a result of the subjective methods used for sampling and categorizing.

Altogether, the samples stretched longitudinally to the direction of muscle fibers had significantly higher tensile strength $(P<0.0001)$, which is in agreement with the findings by Purslow (1985) on the tensile strength of cooked beef. However, comparing each experimental group separately, only the diffuse samples yielded significantly higher tensile strength when they were stretched longitudinal to the fiber direction, compared with the transverse direction of stretching (12.3 vs. $6.02 \mathrm{~N}, P<0.05)$.

Our results showed an increase of tensile strength in diffuse samples by more than $200 \%$ in the cranial area, whereas in the middle area, the increase was approximately $100 \%$. This difference indicates an increased level of woody breast severity in the cranial area compared with the middle area, and previous studies support this result (Petracci et al., 2019). The morphological and gene expression differences related to woody breast in chicken were studied by Clark and Velleman (2016), and they found evidence that the cranial region was more affected than the rest of the muscle. This aspect was also noted in chicken breasts with the white striping condition (Kuttappan et al., 2013).

Offer et al. (1988) mention that the breaking point in unaged beef sternomandibularis muscle is longitudinally $300 \mathrm{kPa}$. The transverse tensile strength in cooked muscle was one-fifth to one-tenth of that, i.e., 30-60 kPa. Buschman et al. (1997) found that frog muscle generates $150-300 \mathrm{kPa}$. The maximum strain generated at contraction or tensile strength in broiler muscle is not well known. Liu et al. (2020), however, studied the force generated by single boiler breast muscle fibers, and on the basis of the results, a maximal force of about $120 \mathrm{kPa}$ can be calculated for fibers of both normal broiler breasts and woody breasts. According to Table 3, the longitudinal values of tensile strength are about $500 \mathrm{kPa}$ or more for normal muscle, but transversally about $250-300 \mathrm{kPa}$, i.e., double the force generated in single fiber tests. Diffuse cases are much stronger. The values given by Offer et al. (1988) were lower, but transverse results were lacking concerning raw meat.

The tensile strength values reported in this paper are mostly expressed as plain newtons, even though the form of force per transverse area (newtons per square millimeter) might have provided more insight on the tensile strength. The dumb-bell shape of the 
Table 3. Tensile strength range of the strips from normal and WB-affected fillets calculated as N/mm $(N=20)$

\begin{tabular}{llccc}
\hline \hline & CL & CT & ML & MT \\
\hline Normal & $0.54-1.91$ & $0.31-1.31$ & $0.99-2.40$ & $0.45-2.04$ \\
Focal WB & $0.83-4.29$ & $0.25-1.74$ & $0.86-2.85$ & $0.53-3.28$ \\
Diffuse WB & $1.78-11.37$ & $0.83-4.93$ & $1.83-8.44$ & $1.02-5.96$ \\
\hline
\end{tabular}

CL, cranial longitudinal; CT, cranial transverse; ML, middle longitudinal; MT, middle transverse; WB, woody breast.

samples was intended to ensure better control over the breaking point, but not all the samples broke at the narrowest point as predicted. It was not possible to control how similar the samples were in relation to muscle fiber bundles or how much fibrotic/nonfibrotic connective tissue there was at the narrowest point of the sample. Therefore, the surface area of the breaking point was not considered, and the results showed a large variation. Samples pulled along the fibers generally had higher tensile strength values/surface area compared with those pulled across the muscle fibers. Tensile strength, expressed as newtons per square millimeter, was calculated at the hypothetical minimum cross sectional area of the sample, and values are shown in Table 3. To our knowledge, no measurements of tensile strength have been performed in previous woody breast studies. Even though these figures do not have a high degree of accuracy, they could still serve as a starting point for future research.

Another aspect that has to be considered is the substantial increase of the range of the values recorded in diffuse samples (Table 3). The cranial area of the chicken breasts seemed to be the most affected by the degree of severity of this condition, according to the mean values, but there were no significant differences $(P>0.05)$. The variation was generally higher in diffuse samples, and the largest variation was in the cranial samples when tension was applied longitudinal to the muscle fiber ( standard deviation $=6.40$ ).

\section{Speculation about links between muscle strength and woody breast syndrome}

Broiler breast muscle is fixed to the keel along its entire proximal side, which does not allow much movement of the muscle of those parts. The birds do not exercise the breast muscles much, and the training activity is not of an aerobic endurance type but short-lived anaerobic fast resistance (strength) activity that is prone to Type IIB fibers (Papinaho et al., 1996; Egan and Zierath, 2013), and the Pectoralis fibers are usually very long in birds (Biewener, 2011). The wings are usually folded down in the resting position in living birds, which means that the sarcomeres in the breast muscle are probably closer to the shorter end of their working range. In a living muscle, the number of sarcomeres is dynamically controlled as follows: if the fibers in a muscle are mostly contracted, the number of sarcomeres will start to decrease, and if extended for a longer period of time, then the number will increase (Goldspink, 1971; Williams and Goldspink, 1971). This results in sarcomere numbers that are dynamically controlled by the slack length of the resting muscle (the so-called resting or slack length is established when the muscle is not active/stretched most of the time). Therefore, the resting length of the sarcomeres should be rather constant irrespective of body size. However, under the current very high growth potential of the breast muscle, this might cause the outer layer-which is less associated with the keel - to grow more than the inner layers that will be limited by dynamic sarcomere length control (slack length). However, there may also be an interaction between age and muscle stretching. Williams and Goldspink (1978) found that the number of sarcomeres was a function of the long-term stretching of muscles of adult rats. However, the same observation was not found in young rats that were still growing. The authors suggested that this may be explained by the lengthening of the tendon. Therefore, a period of fast growth of broilers may not allow balance to be reached in sarcomere length and/or number.

Another aspect is that the force generated by sarcomeres depends on sarcomere length. The further away the sarcomere lengths are from the optimal area of 2.0 $2.5 \mu \mathrm{m}$, the lower the power output is (Gordon et al., 1966; Williams et al., 2013). It would be of interest to know whether the different parts of the broiler breast muscle have different sarcomere lengths in live birds. If so, this will cause differences in force generation; e.g., the innermost sarcomeres may have reached their minimum length while the outermost may still generate strong contraction. However, the dynamic regulation of sarcomere numbers tends to keep the resting length of the sarcomeres constant throughout the muscle. If the moving of wings tends to be painful, the birds will exercise less, and this may stimulate a reduction of sarcomere number and consequently increase the length postmortem.

As mentioned earlier, at rest the wings of living birds are folded down, and the larger downward-driving muscle (Pectoralis major) is shortened and the smaller wing-stretching Supracoracoideus (Pectoralis minor) 
muscle is extended. The extended Deltoid and Supracoracoideus muscle and stretched clavicle bone create a passive tension between upward- and downwardmoving muscles. Because the Pectoralis major is contracted during the majority of the life of the chicken, there is a tendency for the sarcomere numbers to be reduced, due to the dynamic drive in muscle to the constant resting length of the sarcomeres. This may then mean that, if wing flapping is necessary, the operational length of sarcomeres will not be optimal in woody breast muscles. However, as Biewener (2011) mentioned, Pectoralis muscle has a special property of creating force within a long contraction distance from their resting length. Our hypothesis to which this study gives some basic information is that the force during wing flapping may cause friction between fibers as well as between muscle fiber bundles. Further studies are ongoing to study this aspect histologically as well as having the third dimension for the tensile strength: from the surface inward. The muscle fiber bundles in breast muscles are especially bent in the cranial end, and because the fibers are long, they are bent as well. When the muscle fiber bundle contracts, the outer part of it contracts more than the inner part, so there will be some tension between the bundles above and below the contracting bundle. Further studies will be needed to evaluate the effect of rapidly increasing the size of breast muscles, and thus curved fibers, on possible intramuscle tensions in order to shed some light on the onset of spaghetti meat, in which a clear separation of layers can be seen in transverse cut.

\section{Conclusions}

Woody breast muscles have markedly longer sarcomere lengths compared with normal muscles, if electrical stimulation is not used. The difference in sarcomere length between woody breast and normal breast is manifested in the surface layer of the muscle and not in the deeper layer. Future studies should also investigate the caudal area of the Pectoralis muscle in order to obtain a more complete profile of sarcomere lengths in woody breast. This study showed no significant difference between the mean values of tensile strength in normal samples and focal woody breast samples, but the diffuse samples yield significantly higher tensile strength, especially when stretched longitudinal to muscle fiber direction. In light of the current findings on tensile strength, the force production capability of woody breast muscles should be investigated to test the hypothesis about whether mechanical strains have an effect on the formation of muscle syndromes, e.g., woody breast or spaghetti meat. In conclusion, although this study did not show the sarcomere lengths in living muscle, it suggests an imbalance in sarcomere length in different parts of the breast muscle, which may induce a reduction in the functionality and strength of the muscle.

Data availability statement: All data supporting the conclusions in this study are available from the corresponding authors upon request by qualified researchers.

\section{Acknowledgments}

The authors want to thank Kaisa Korteniemi, PhD, for doing the statistical calculations and assisting in methods training. The University of Helsinki and Erasmus Mundus Food of Life Educational Program are greatly acknowledged for providing funding. Author contributions: All authors have made a substantial and intellectual contribution to the work and approved it for publication. TEJP and CGC planned the experiments and designed the study. CGC performed the laboratory analysis. All authors participated in interpretation of data and writing the manuscript. Conflict of interest statement: The authors declare that the submitted work was conducted in the absence of any personal, commercial, or financial relationships that could potentially be construed as a conflict of interest.

\section{Literature Cited}

Abasht, B., M. F. Mutryn, R. D. Michalek, and W. R. Lee. 2016. Oxidative stress and metabolicperturbations in wooden breast disorder in chickens. PLoS ONE. 11:e0153750. https://doi. org/10.1371/journal.pone.0153750.

Ashmore, C., K. Mechling, and Y. Lee. 1988. Sarcomere length in normal and dystrophic chick muscles. Exp. Neurol. 101:221227. https://doi.org/10.1016/0014-4886(88)90005-2.

Baldi, G., F. Soglia, M. Mazzoni, F. Sirri, L. Canonico, E. Babini, L. Laghi, C. Cavani, and M. Petracci. 2018. Implications of white striping and spaghetti meat abnormalities on meat quality and histological features in broilers. Animal. 12:164-173. https://doi.org/10.1017/S1751731117001069.

Biewener, A. A. 2011. Muscle function in avian flight: achieving power and control. Philos. T. R. Soc. B. 366:1496-1506. https://doi.org/10.1098/rstb.2010.0353.

Buschman, H. P., W. J. van der Laarse, G. J. Stienen, and G. Elzinga. 1997. Variation in normalized isometric tetanic force of isolated fast-twitch muscle fibres of Rana temporaria. J. Exp. Biol. 200:523-529.

Clark, D. L., and S. G. Velleman. 2016. Spatial influence on breast muscle morphological structure, myofiber size, and gene expression associated with the wooden breast myopathy in 
broilers. Poultry Sci. 95:2930-2945. https://doi.org/10.3382/ ps/pew243.

Chatterjee, D., H. Zhuang, B. C. Bowker, A. M. Rincon, and G. Sanchez-Brambila 2016. Instrumental texture characteristics of broiler pectoralis major with the wooden breast condition. Poultry Sci. 95:2449-2454. https://doi.org/10.3382/ps/ pew204.

Cross, H. R., R. L. West, and T. R. Dutson. 1981. Comparison of methods for measuring sarcomere length in beef semitendinosus muscle. Meat Sci. 5:261-266. https://doi.org/10.1016/ 0309-1740(81)90016-4.

Dalle Zotte, A., M. Cecchinato, A. Quartesan, J. Bradanovic, G. Tasoniero, and E. Puolanne. 2014. How does "wooden breast" myodegeneration affect poultry meat quality? Proc. 60th Int. Congr. Meat Sci. Technol, Punta del Este, Uruguay. Session 8, 25.

Egan, B., and J. R. Zierath. 2013. Exercise metabolism and the molecular regulation of skeletal muscle adaptation. Cell Metab. 17:162-184. https://doi.org/10.1016/j.cmet.2012.12. 012.

Ertbjerg, P., and E. Puolanne. 2017. Muscle structure, sarcomere length and influences on meat quality: A review. Meat Sci. 132:139-152. https://doi.org/10.1016/j.meatsci.2017.04.261.

Goldspink, G. 1971. Changes in striated muscle fibres during contraction and growth with particular reference to myofibril splitting. J. Cell Sci. 9:123-137.

Gordon, A. M., A. F. Huxley, and F. J. Julian. 1966. Variation in isometric tension with sarcomere length in vertebrate muscle fibres. J. Physiol.-London. 184:170-192. https://doi.org/10. 1113/jphysiol.1966.sp007909.

Kang, I. S., and A. R. Sams. 1999. Bleedout efficiency, carcass damage, and rigor mortis development following electrical stunning or carbon dioxide stunning on a shackle line. Poultry Sci. 78:139-143. https://doi.org/10.1093/ps/78.1.139.

Kuttappan, V. A., H. L. Shivaprasad, D. P. Shaw, B. A. Valentine, B. M. Hargis, F. D. Clark, S. R. McKee, and C. M. Owens. 2013. Pathological changes associated with white striping in broiler breast muscles. Poultry Sci. 92:331-338. https:// doi.org/10.3382/ps.2012-02646.

Li, C., P. Shi, C. Xu, X. Xu, and G. Zhou. 2010. Tracing processes of rigor mortis and subsequent resolution of chicken breast muscle using a texture analyzer. J. Food Eng. 100:388-391. https://doi.org/10.1016/j.jfoodeng.2009.10.040.

Liu, J., E. Puolanne, Schwartzkopf, A. Arner. 2020. Altered sarcomeric structure and mechanics in woody breast myopathy in avian pectoralis major. Front Physiol. 11:287. https://doi.org/ 10.3389/fphys.2020.00287.

Liu, J., M. Ruusunen, E. Puolanne, and P. Ertbjerg. 2014. Effect of pre-rigor temperature incubation on sarcoplasmic protein solubility, calpain activity and meat properties in porcine muscle. LWT-Food Sci. Technol. 55:483-489. https://doi. org/10.1016/j.lwt.2013.10.001.

Norring, M., A. Valros, J. Valaja, H-K. Sihvo, Immonen, K., and E. Puolanne. 2019. Wooden breast myopathy links with poorer gait in broiler chickens. Animal. 13:1690-1695. https://doi. org/10.1017/S1751731118003270.

Offer, G., P. Purslow, R. Almond, T. Cousins, J. Elsey, G. Lewis, N. Parsons, and A. Sharp. 1988. Myofibrils and meat quality.
Proc. 34th Int. Congr. Meat Sci. Technol., Brisbane, Australia, 29 August-2 September 1988. Session 4, 2.

Papa, C. M., and D. I. Fletcher. 1988. Pectoralis-muscle shortening and rigor development at different locations within the broiler breast. Poultry Sci. 67:635-640. https://doi.org/10.3382/ps. 0670635.

Papah, M. B., E. M. Brannick, C. J. Schmidt, and B. Abasht. 2017. Evidence and role of phlebitis and lipid infiltration in the onset and pathogenesis of Wooden Breast Disease in modern broiler chickens. Avian Pathol. 46:623-643. https://doi.org/10.1080/ 03079457.2017.1339346.

Papinaho, P. A., M. Ruusunen, T. Suuronen, and D. L. Fletcher. 1996. Relationship between muscle biochemical and meat quality properties of early deboned broiler breasts. J. Appl. Poultry Res. 5:126-133. https://doi.org/10.1093/japr/5.2.126.

Petracci, M., F. Soglia, M. Madruga, L. Carvalho, I. Elza, and M. Estévez. 2019. Wooden-breast, white striping, and spaghetti meat: Causes, consequences and consumer perception of emerging broiler meat abnormalities. Compr. Rev. Food Sci. F. 18:565-583. https://doi.org/10.1111/1541-4337.12431.

Purslow, P. 1985. The physical basis of meat texture - observations on the fracture-behavior of cooked bovine M-semitendinosus. Meat Sci. 12:39-60. https://doi.org/10.1016/0309-1740(85) 90024-5.

Ramaswamy, K. S., M. L. Palmer, J. H. van der Meulen, A. Renoux, T. Y. Kostrominova, D. E. Michele, and J. A. Faulkner. 2011. Lateral transmission of force is impaired in skeletal muscles of dystrophic mice and very old rats. J. Physiol.-London. 589:1195-1208. https://doi.org/10.1113/ jphysiol.2010.201921.

Sams, A. R., and C. S. Dzuik. 1999. Meat quality and rigor mortis development in broiler chickens with gas- induced anoxia and postmortem electrical stimulation. Poultry Sci. 78:14721476. https://doi.org/10.1093/ps/78.10.1472.

Sihvo, H-K., N. Airas, J. Lindén, and E. Puolanne. 2018. Pectorial vessel density and early ultrastructural changes in broiler chicken wooden breast myopathy. J. Comp. Pathol. 161:110. https://doi.org/10.1016/j.jcpa.2018.04.002.

Sihvo, H-K., K. Immonen, and E. Puolanne. 2014. Myodegeneration with fibrosis and regeneration in the pectoralis major muscle of broilers. Vet. Pathol. 51:619-623. https://doi.org/10.1177/ 0300985813497488.

Soglia, F., J. Gao, M. Mazzoni, E. Puolanne, C. Cavani, M. Petracci, and P. Ertbjerg. 2017. Superficial and deep changes of histology, texture and particle size distribution in broiler Wooden Breast muscle during refrigerated storage. Poultry Sci. 96:3465-3472. https://doi.org/10.3382/ps/pex115.

Soglia, F., S. Mudalal, B. Babini, M. Di Nunzio, M. Mazzoni, F. Sirri, C. Cavani, and M. Petracci. 2016. Histology, composition, and quality traits of chicken muscle affected by wooden breast abnormality. Poultry Sci. 95:651-659. https://doi.org/ $10.3382 / \mathrm{ps} /$ pev353.

Soglia, F., M. Petracci, and E. Puolanne. 2019. Sarcomere lengths in wooden breast broiler chickens. Ital. J. Anim. Sci. 19:569572. https://doi.org/10.1080/1828051X.2020.1761271.

Sun, X., D. A. Koltes, C. N. Coon, K. Chen, and C. M. Owens. 2018. Instrumental compression force and meat attribute changes in woody broiler breast fillets during short-term 
storage. Poultry Sci. 97:2600-2606. https://doi.org/10.3382/ ps/pey107.

Tijare, V. V., F. L. Yang, V. A. Kuttappan, C. Z. Alvarado, C. N. Coon, and C. M. Owens. 2016. Meat quality of broiler breast fillets with white striping and woody breast muscle myopathies. Poultry Sci. 95:2167-2173. https://doi.org/10.3382/ps/pew129.

Velleman, S. G., D. L. Clark, and J. R. Tonniges. 2018. The effect of wooden breast myopathy on sarcomere structure and organization. Avian Dis. 62:28-35. https://doi.org/10.1637/ 11766-110217-Reg.1.
Williams, P. E., and G. Goldspink. 1971. Longitudinal growth of striated muscle fibres. J. Cell Sci. 9:751-767.

Williams, P. E., and G. Goldspink. 1978. Changes in sarcomere length and physiological properties in immobilized muscle. J. Anat. 127:459-468.

Williams, C. D., M. K. Salcedo, T. C. Irving, M. Regnier, and T. L. Daniel. 2013. The length-tension curve in muscle depends on lattice spacing. Proc. R. Soc. B. 280:20130697. https://doi. org/10.1098/rspb.2013.0697. 